\section{GERIATRIC ONCOLOGY}

Syed Md Akram Hussain ${ }^{1}$, Shah Md Keramat $\mathrm{Ali}^{2}$, Kazi Manzur Kader ${ }^{3}$

\begin{abstract}
:
We are growing old. In cancer care, there is no exception. Over one-half of all cancer patients in the United States are over age 60 .Elderly represent $12 \%$ of the total world population. Over the next 25 years, more than $60 \%$ of all cancers diagnosed in people of 55 years old and older in Bangladesh As the number of elderly rise exponentially, we can expect growing numbers of elderly at risk for developing cancer. Without dramatic breakthroughs in cancer prevention and treatment, it will have a major impact on the magnitude of cancer problem. In the meantime, when we are growing old and facing cancer, several important realities need to address.
\end{abstract}

What is most important to consider in treatment planning for cancer, is not what the patient's age is, but rather about the baseline level of function and fitness despite chronologic age. In the absence of co-morbid conditions, advanced age should not be a deterrent to cancer therapy with a curative intent. Without other chronic illness which states impair kidney, liver, lung and heart function, older patients can expect comparable results to therapeutic regimens as their younger counterparts.

\section{Key word: Geriatric Oncology}

Whether we do not want it or not, but we are growing old. In the specialty of cancer care, there is no exception to this trend. Over one-half of all cancer patients in the United States are over age 60 . What is even more significant is that the elderly represent $12 \%$ of the total population. Over the next 25 years, more than $60 \%$ of all cancers diagnosed in people of 55 years old and older in Bangladesh. Without dramatic breakthroughs in cancer prevention and treatment, the graying of world will have a major impact on the magnitude of the cancer problem. As the number of elderly rise exponentially, we can expect growing numbers of elderly at risk for developing cancer. In the meantime, when we are growing old and facing cancer, several important realities need to address.

There has been widespread acceptance that chronologic age equates with physiological age and hence the elderly, with their projected physiological decline, could not withstand the rigors of aggressive cancer therapies. Fortunately, there is newfound attention to the fact that this parallel is indeed an erroneous one. Physiologic age is distinct from chronologic

1. Professor and Chairman, Department of Oncology, Bangabandhu Sheikh Mujib Medical University

2. Professor, Institute of Nutrition and Food Science, University of Dhaka

3. Associate Professor, Radiation Oncology, National Institute of Cancer Research \& Hospital age. A 70-year-old man could indeed have the physiologic reserve and stamina of a 55-year-old. What is most important to consider in treatment planning for cancer, is not what the patient's age is, but rather about the baseline level of function and fitness despite chronologic age. In the absence of comorbid conditions, advanced age should not be a deterrent to cancer therapy with a curative intent. Without other chronic illness which states impair kidney, liver, lung and heart function, older patients can expect comparable results to therapeutic regimens as their younger counterparts.

First, there is little recognition that cancer is indeed a disease of aging. Despite the prominence of cancer in the elderly, there is no such special care is available in Bangladesh. They are no dedicated and committed force devoted for the elderly. Is our bias showing? Do we consider the loss of the old? Second, up until the end of this past millennium, the elderly have excluded from entry into clinical trials, thereby precluding study of their response to a wide range of cancer therapies.

The decision to exclude older patients from clinical trials based on assumption. We need an 'attitude adjustment' to jumpstart a much-needed initiative, which focuses research, education, and clinical innovation on the forgotten majority. Ageism or societal prejudice towards the elderly need to be confront with extreme urgency in cancer care. Our patients deserve our utmost attention as we mature in tandem.

Despite the prevalence of cancer in the elderly, pain is more likely to be unrecognized and untreated. Collaboration to optimize geriatricians, oncologists and palliative care specialists for pain assessment and management in older patients, whether treated at home or in a long-term care facility is very important. Here we will discuss about tools, resources and research evidence to enhance the quality of care of pain in older patient.

\section{Diagnosing Pain in the Older Patient}

Pain does not have to come with aging. Simple strategies can uncover pain that can be relieved in many patients who may be used to putting up with it. Research has shown that as people age, they are more and more reticent to report pain, and they are reluctant to label a sensation as pain. Sometimes seniors hesitant to report their pain. This reluctance may stem from the belief that pain is to be expected and tolerated; or from the belief that pain indicates a new life-threatening disease, or that they will become dependent on pain medications. We could diagnose pain in the older person by asking about aching, soreness, discomfort and not just pain, since this word is loaded for elders. Ask the patient how they feel here and now. Open-ended questions help make the patient comfortable with discussing fear, anxiety and other non-physical symptoms. It is very important to diagnose suffering because relief of pain goes hand in hand with treatment of the emotional and spiritual components that contribute to it. 
American Geriatrics Society guidelines recommend approaching patients with a standard 10-point pain scale before moving to alternative scales such as pain thermometers. The most important step is to make sure to use a scale the patient understands. We know that the simple use of pain assessment scales with elderly nursing home residents greatly increases the frequency of diagnosing pain, especially among the oldest patients (>85 years old). When verbal communication is possible, older patients can rate their pain on their own. The choice of self-rating scale depends on the cognitive ability of the patient, with simple scales more likely to complete than multidimensional scales. Practically, the clinician should have a range of scales available when assessing older people.

A geriatric assessment is a fundamental screening tool in caring for the elderly. It is useful to estimate life expectancy and tolerance of treatment and to identify factors that may interfere with cancer therapy, including depression, malnutrition, anemia, neutropenia and lack of caregiver support. A geriatric assessment can help to determine the best use of supportive care agents and identify frail patients for whom palliative care is the best option. Life expectancy is critical to estimate the risks and benefits of pharmacologic management, such as the choice of an opioid route of administration: while intrathecal infusion would tolerate in an otherwise healthy elderly, the oral or transdermal route may be better suited to a frail senior.

Pain is a reflection of disease severity. We know from recent research that older cancer patients with three or more coexisting illnesses have increased pain. Age-in and of itself- influences the experience of pain for patients with advanced cancer because the prevalence of painful conditions increases dramatically in later life. Some therapy-related pain, such as the painful neuropathy associated with chemotherapy, affects the elderly disproportionately. Psychological problems such as anxiety, depression and delirium, social factors such as concerns about leaving loved family members, and spiritual concerns, such as the meaning of one's life, and issues of guilt, love, and forgiveness also affect the pain experience.

\section{Beliefs And Attitudes of Elderly Patients Known to Add Complexity to Pain Management}

Elders refuse treatment sometimes -that they do not understand; that is too expensive; that causes unacceptable side effects.Elders believe pain is a normal part of aging. Elders are more stoic and reticent than younger adults to communicate pain. Elders believe using any medication daily is a sign of addiction. Elders are physiologically more sensitive to medications.Elderly patients with cancer may have multiple sources of pain: underlying chronic non-malignant pain that may predate the cancer, pain from the malignancy, and pain from the treatment. It is also important to assess for depression, delirium, and sources of social and spiritual distress.
Recent work shows exacerbation of pain and suffering when older patients have several diseases across multiple organ systems. This demonstrates that evaluation of the presence of comorbidity should be a part of the routine assessment of all older pain patients. There is evidence that chronic pain is a significant predictor of loss in function in elders, independently of the patient's treatment or of any coexisting disease. Since older patients have limited reserves, pain can have long-lasting and irreversible consequences that compromise the cancer therapy, the function and even the survival of the patient. The possibility that pain may limit movement and induce depression or poor nutrition is of special concern in older adults.

Diagnosing Pain in the Older Patient with Cognitive Impairment In this case, physicians must ask caregivers about behavioral changes. Frowns, grimacing during movement, body guarding commonly accompany pain. In fact any behavioral change is a sign of trouble in this patient population, so it is important to know what baseline routine behaviors are. Refusing food, difficulty sleeping, increased wandering are all examples of behavior changes that should be monitored.Scales and protocols have been developed to assess discomfort in residents of long-term care facilities who are no longer able to report pain because of dementia.

Reliable pain assessment can be obtained from a patient with a failing memory. Clinicians and caregivers must believe patients first and take their report of pain seriously. It would be inappropriate to consult a family member or another caregiver rather than the patient. An excellent strategy consists of combining behavioral observation with a pain interview and the use of a pain scale. Vertical visual scales, such as pain thermometers, and numerical or verbal descriptor scales are most suitable for older adults with cognitive impairments and/ or a low educational level.

\section{Managing Pain in The Older Cancer Patient}

Aging has important effects on how the body handles drugs. Changes in body composition, metabolic rate, hepatic mass, blood flow and glomerular filtration rate affect how the body absorbs, distributes and eliminates chemicals. Because older patients take more medications, there is an increasing risk of toxicity and of drug-drug interactions when managing their pain. Also, the elderly are less able to clear most analgesic and adjuvant medications.

The WHO three-step analgesic ladder is a useful guide to prescribing for older patients. A basic principle for relieving pain in the elderly is to start analgesics at low doses and titrate them slowly while monitoring their effects. As a rule, analgesics are safe to use in older people without overt liver and renal disease, provided you use the lowest dose compatible with functional improvement; it is important to remember that the goal of pain therapy is to maintain optimum function and quality of life.Age-related factors can contribute to longer duration of 
opioid action in the older adult. Elderly patients experience opioid side effects more readily than younger patients. The gastrointestinal side effects of opioids and NSAIDs, such as dry mouth, nausea and constipation can turn some patients away from the benefits of these agents.

\section{Skills Necessary to Optimize Pain Control in Older Cancer Patients}

- ability to objectively assess the functional age of a patient (not necessarily related to chronological age since the rate of decline varies);

- ability to understand the impact of coexisting conditions;

- ability to titrate numbers and types of drugs taken at the same time (polypharmacy);

- knowledge of the biology of aging;

- ability to be patient and to listen to the patient;

- knowledge to make the patient central to pain assessment.

The patient should be started on a potent analgesic such as morphine in case of severe pain. There is no reason to delay and find out that milder analgesics don't work in a patient with obvious severe pain. It is best to use the least invasive route of administration in the elderly. Older people are more susceptible to the neuropathic complications of cancer therapy, especially radiation and chemotherapy. They also may suffer from diabetic neuropathies or post-herpetic neuralgia. Anticonvulsants and tricyclic antidepressants are useful agents to manage neuropathic pain in this population.Nonpharmacological management such as massage, heat/cold are used successfully. Cognitive-behavioral therapies that enhance coping have been very successful in randomized controlled trials. Maintaining communication with cancer pain patients and their families can decrease anxiety which exacerbates the pain experience.

Pain is influenced by the response of partners and caregivers. Patients living at home, as well as patients in residential care settings rely upon family and friends to advocate for them. We know that when spouses misjudge the patient's pain or lack confidence in their ability to help the patient control pain, patients report more pain and distress.Most important aspect of pain management in older people is assessment. After assessing pain and identifying the goals of care, it is best to simplify drug regimens as much as possible with the elderly. Lastly, combining drug and non-drug interventions is the most effective pain therapy for older adults.

\section{References:}

1. Crivellari D, Bonetti M, Castiglione-Gertsch $\mathrm{M}$ et al. Burdens and benefits of adjuvant cyclophosphamide, methotrexate, and fluorouracil and tamoxifen for elderly patients with breast cancer: The International Breast Cancer Study Group Trial VII. J Clin Oncol 2000; 18: 1412-1422.

2. Extermann M, Aapro M. Assessment of the older cancer patient. Hematol Oncol Clin North Am 2000; 14: 63-77.

3. Lichtman S, Skirvin JA, Vemulapalli S. Pharmacology of antineoplastic agents in older cancer patients. Crit Rev Oncol Hematol 2003; 46: 101-114.

4. International Breast Cancer Study Group (IBCSG). Endocrine responsiveness and tailoring adjuvant therapy for postmenopausal lymph node-negative breast cancer: a randomized trial. J Natl Cancer Inst 2002; 94: 1054-1065.

5. Fargeot P, Roche H, Bonneterre JM et al. Disease-free survival (DFS) advantage of weekly epirubicin plus tamoxifen vs tamoxifen (Tam) alone as adjuvant treatment of operable, node-positive $(\mathrm{N}+$ ) elderly breast cancer (BC) patient (pts): 5 year-follow-up results of French adjuvant study group, FASG-08 trial. Proc Am Soc Clin Oncol 2002; 21: 37a (Abstr 145).

6. Parkin DM. Global cancer statistics in the year 2000. Lancet Oncol 2001; 2: 533-543.

7. Alberg AJ, Singh S. Epidemiology of breast cancer in older women: implications for future healthcare. Drugs Aging 2001; 18: 761-772.

8. National Cancer Institute. Facts and figures about cancer clinical trials. http://www.nci.nih.gov/clinicaltrials/factsand-figures

9. Repetto L, Fratino L, Audisio RA et al. Comprehensive geriatric assessment adds information to Eastern Cooperative Oncology Group performance status in elderly cancer patients: An Italian Group for Geriatric Oncology study. J Clin Oncol 2002; 20: 494-502.

10. Katz S, Downs TD, Cash HR, Grotz RC. Progress in development of the index of ADL. Gerontologist 1970; 10:20-30.

11. Lawton MP, Brody EM. Assessment of older people: selfmaintaining and instrumental activities of daily living. Gerontologist 1969; 9: 179-186.

12. Folstein MF, Folstein SE, McHugh PR. "Mini-Mental State”. A practical method for grading the cognitive state of patients for the clinician. J Psychiatr Res 1975; 12: 189-198.

13. Zagonel V, Fratino L, Piselli P et al. The comprehensive geriatric assessment (CGA) predicts mortality among elderly cancer patients (ECP). Proc Am Soc Clin Oncol 2002; 21: 365a (Abstr 1458). 